\title{
AS AÇÕES DE ENFERMAGEM NA ATENÇÃO BÁSICA DIANTE DOS RISCOS DA HIPERTENSÃO ARTERIAL NA GESTAÇÃO
}

\section{ARTIGO DE REVISÃO}

ADRIÃO, Iracely Santos ${ }^{1}$, BARBOSA, Marluce Sampaio Nobre ${ }^{2}$

ADRIÃO, Iracely Santos. BARBOSA, Marluce Sampaio Nobre. As ações de enfermagem na Atenção Básica diante dos riscos da hipertensão arterial na gestação. Revista Científica Multidisciplinar Núcleo do Conhecimento. Ano 06, Ed. 09, Vol. 04, pp. 84-100. Setembro de 2021. ISSN: 2448-0959, Link de acesso: https:/www.nucleodoconhecimento.com.br/saude/riscos-da-hipertensao, DOI: 10.32749/nucleodoconhecimento.com.br/saude/riscos-da-hipertensao

\section{RESUMO}

A pré-eclâmpsia se apresenta como uma das síndromes hipertensivas que podem acometer mulheres em período de gravidez, durante o parto e/ou no período de pósparto até 10 dias, de forma que ainda se constitui como uma patologia que quando não adequadamente diagnosticada e tratada, pode causar grandes prejuízos e até levar à morte da gestante e/ou parturiente. Este estudo tem por objetivo principal conhecer as condutas de prevenção da pré-eclâmpsia na atenção básica pela enfermagem. Portanto, tem como questão norteadora: quais as ações de enfermagem na Atenção Básica diante dos riscos da hipertensão arterial na gestação? O presente trabalho se fundamenta em uma pesquisa bibliográfica, com abordagem qualitativa realizada por meio do método da revisão integrativa. Para coletar os dados foi realizada uma investigação nas bases de dados: Google Acadêmico, Pubmed, Scielo e Medline. Os descritores utilizados foram: Riscos. Gestação. Hipertensão Arterial. Enfermagem. Os critérios de inclusão utilizados para a seleção da amostra foram: artigos disponíveis eletronicamente publicados no

\footnotetext{
${ }^{1}$ Graduanda em Enfermagem pela Universidade CEUMA.

${ }^{2}$ Mestrado em Doenças Tropicais.
}

RC: 97235

Disponível em: https://www.nucleodoconhecimento.com.br/saude/riscos-dahipertensao 
período de 2015 até 2020; idioma português. Os critérios de exclusão foram artigos inferiores ao ano de 2015 e palavras-chaves que não estejam relacionados ao tema. Para a análise na íntegra dos artigos selecionados, foi utilizado plataforma online (busca em bases de dados), com o propósito de extrair (título e objetivo dos artigos), organizar (em forma de tabela), sumarizar as informações e facilitar a formação do banco de dados. Os resultados apontaram 35 artigos publicados, mas apenas 15 artigos, dos últimos cinco anos, têm como temática principal a prevenção e os riscos da pré-eclâmpsia na atenção básica. Conclui-se que as principais condutas utilizadas não somente pelo enfermeiro, mas por toda a equipe atuante na atenção básica, devem se pautar no processo de acolhimento voltado para as ações que podem ser determinantes na prevenção da pré-eclâmpsia.

Palavras-chave: Riscos, Gestação, Hipertensão Arterial, Enfermagem.

\section{INTRODUÇÃO}

Vive-se atualmente em um mundo marcado profundamente pela produção de saberes em todas as áreas, incluindo a enfermagem e a medicina. Mesmo com isso ainda é muito grande o número de mulheres acometidas por doenças amplamente conhecidas, como a Síndrome Hipertensiva Especifica da Gestação (SHEG). Sua incidência é alta, a ponto de ser tratada como problema de saúde pública (ARAÚJO, et al, 2017).

Se as síndromes hipertensivas ligadas ao estado de gestação das mulheres se mostram tão importantes, portanto, devem ser de conhecimento das autoridades e dos profissionais de saúde, e por isso é de se perguntar, porque em pleno século $\mathrm{XXI}$, tais patologias ainda comprometem a saúde e muitas vezes até a vida da gestante e dos neonatos. Quando se sabe que a prática de exames pré-natais identificaria o problema, ou pelo menos permitiria seu diagnóstico precoce, o que, na prática permitiria uma intervenção em tempo hábil (ARNALDO; CARDOZO, 2021). 
Todas essas reflexões contribuíram e motivaram para a escolha do tema como objeto de estudo, para sua viabilização levou-se em parte o seguinte problema: temse visto um elevado índice de gestantes que são atendidas quase sempre apresentando sinais e sintomas de SHEG, principalmente pré-eclâmpsia. Então, quais as ações de enfermagem na Atenção Básica diante dos riscos da hipertensão arterial na gestação? E como resposta provisória ao problema enunciado, adotou-se a hipótese de que às mulheres em período gestacional e mesmo parturientes e no período pós-parto, apresentam alto índice de pré-eclâmpsia, porque não tiveram o adequado acompanhamento pré-natal (ARAÚJO et al., 2017).

Estudos que envolvem esse conjunto de informações (riscos da hipertensão arterial na gestação), auxiliam na prevenção da hipertensão, haja vista que, estimula e alerta a população a adotar mudanças nos hábitos alimentares e na prática habitual da atividade física, bem como facilita a abordagem medicamentosa de seus componentes isolados ou da própria síndrome metabólica, por meio de profissionais e pesquisadores (ARNALDO; CARDOZO, 2021).

A escolha do tema atua para finalidade de trazer para as mulheres grávidas informações que as levem a ter uma vida mais saudável, pois essa é a tarefa principal dos profissionais de saúde. E para estas mulheres no período de gestação devem começar levar para seu dia a dia uma alimentação mais saudável e natural, tendo como aconselhamento o consumo de frutas, verduras, legumes. Sendo orientadas a minimizar o consumo de carnes, gorduras, frituras, sanduiches e outras formas de alimentação prejudicial à saúde e que leva ao aumento da hipertensão arterial (SOARES et al., 2015).

Para uma boa qualidade de vida, a mulher grávida considerado hipertensa precisa manter uma vida saudável, pois muitos destas aderem ao tratamento farmacológico, onde a pressão arterial é controlada através de ingestão de comprimidos. Existe também o tratamento não-farmacológico que é realizado através de práticas de atividade física, que pode ser também um complemento junto ao tratamento farmacológico (MELO et al., 2015). 
A prática de exercício é de fundamental importância para a saúde e qualidade de vida de modo em geral e principalmente para a mulher gravida, que tem o hábito de levar uma vida sedentária, já que está no período de descanso da profissão, e começa o sedentarismo (SOARES et al., 2015).

O trabalho se justifica por ser de grande interesse para a enfermagem e a mulher que pretende ser mãe. $O$ interesse pela pesquisa também foi motivado devido este ser um tema que traz importante e fundamental informação para toda mulher que tem objetivo de engravidar e para a área de atuação da enfermagem.

E assim a pesquisa traz ensinamentos para uma boa qualidade de vida da mulher grávida, uma vez que a hipertensa precisa manter uma vida saudável, pois muitos destas aderem ao tratamento farmacológico, onde a pressão arterial é controlada através de ingestão de comprimidos. Existe também o tratamento não-farmacológico que é realizado através de práticas de atividade física, que pode ser também um complemento junto ao tratamento farmacológico (SANTOS; NETO, 2016).

O objetivo principal desse trabalho consiste em principal conhecer as condutas de prevenção da pré-eclâmpsia na atenção básica pela enfermagem.

\section{METODOLOGIA}

Quanto aos procedimentos metodológicos do presente estudo foi utilizada o desenvolvimento da pesquisa bibliográfica, ou seja, revisão de literatura de artigos científicos sobre o tema Hipertensão e Gestação. Para a presente pesquisa científica realizou-se a busca dos artigos nas bases de dados do Google Acadêmico, Pubmed, Scielo e Medline, revistas de saúde livros e artigos científicos, com bases em materiais já elaborado.

Além disso, a pesquisa exploratória, para Lakatos e Marconi (2010, p. 171) "os estudos exploratório-descritivo combinados tem por objetivos descrever completamente determinado fenômeno, como, por exemplo, o estudo de um caso para o qual são realizadas análises empíricas e teóricas".

RC: 97235

Disponível em: https://www.nucleodoconhecimento.com.br/saude/riscos-dahipertensao 
Portanto, a pesquisa busca sempre a melhor aproximação do pesquisador com a situação real dos problemas, além disso, consiste na compreensão de maneira menos totalitária, fragmentando as opiniões apresentadas e objetivando a perspectiva da ótica dos atores que estão envolvidos no processo.

Em relação aos meios, foi aplicado para este estudo os procedimentos de pesquisa bibliográfica. Assim, publicações nacionais, em livros, artigos de autoria de profissionais de enfermagem e revistas farão parte da construção desse material (FONSECA, 2002).

Portanto, é perceptível que, apesar de todo o esforço para levantar os pontos de vista dos atores sociais envolvidos no processo ao qual a pesquisa se propõe a estudar, tem-se que o embasamento teórico através do levantamento bibliográfico, se torna o alicerce para a compreensão da pesquisa.

Nesse estudo foi levantado o entendimento de alguns autores com base na pesquisa bibliográfica detalhadamente em busca da visão sobre 0 referido estudo. Enriquecendo em conhecimento, pois é de fundamental importância verificar o posicionamento desses autores a respeito da enfermagem e os riscos da hipertensão arterial na gestação.

Portanto, os critérios de inclusão foram: artigos publicados em língua portuguesa, que aborde a enfermagem e os riscos da hipertensão arterial na gestação, e que tenham sido publicados e indexados nos referidos bancos de dados de 2015 até 2020. Os critérios de exclusão foram artigos inferiores ao ano de 2015 e palavraschaves que não estejam relacionados ao tema. As palavras chaves utilizadas foram: Riscos. Gestação. Hipertensão Arterial. Enfermagem. A coleta de dados também foi realizada no primeiro semestre de 2021.

A presente pesquisa teve entre suas fontes de coleta de dados, mediante catalogação e fichário de documentação bibliográfica, livros, artigos, revistas, jornais, periódicos, e matérias veiculadas na Internet, de autores contemporâneos, por meio dos quais foram realizadas diversas interpretações do tema. Assim, é de

RC: 97235

Disponível em: https://www.nucleodoconhecimento.com.br/saude/riscos-dahipertensao 
relevância mencionar, os meios de coleta de dados relacionados, é o que se espera para cultivar da melhor forma possível as origens e as motivações das respostas encontradas ou não ao final da pesquisa.

\section{DESENVOLVIMENTO}

A amostra final desta revisão foi constituída por 15 (quinze) artigos científicos, selecionados pelos critérios de inclusão previamente estabelecidos. O quadro 1 representa as especificações de cada um dos artigos, distribuídos, segundo: ano; periódico; nome dos autores e título.

Quadro 1. Relação dos estudos selecionados quanto ao ano, periódico, autores e título entre 2015 e 2020.

\begin{tabular}{|c|c|c|c|}
\hline ANO & PERIÓDICO & AUTORES & TÍTULO \\
\hline 2017 & $\begin{array}{l}\text { Rev enferm UFPE } \\
\text { on line }\end{array}$ & $\begin{array}{l}\text { ARAÚJO, Isabella } \\
\text { Félix Meira, et al }\end{array}$ & $\begin{array}{l}\text { Síndromes hipertensivas e } \\
\text { fatores de risco associados à } \\
\text { gestação }\end{array}$ \\
\hline 2021 & $\begin{array}{l}\text { Revista científica } \\
\text { multidisciplinar } \\
\text { núcleo do } \\
\text { conhecimento }\end{array}$ & $\begin{array}{lr}\text { ARNALDO, } & \text { Mariany } \\
\text { de } & \text { Freitas. } \\
\text { CARDOZO, Maryanne } \\
\text { Neuraide Freire }\end{array}$ & $\begin{array}{l}\text { Assistência de enfermagem } \\
\text { às gestantes hipertensas na } \\
\text { prevenção da prematuridade: } \\
\text { revisão bibliográfica }\end{array}$ \\
\hline 2019 & $\begin{array}{l}\text { Trabalho } \\
\text { Conclusão } \\
\text { Curso }\end{array}$ & $\begin{array}{l}\text { CABRAL, } \\
\text { Pereira }\end{array}$ & $\begin{array}{l}\text { Cuidados de enfermagem a } \\
\text { mulheres com doença } \\
\text { hipertensiva específica da } \\
\text { gestação: uma revisão } \\
\text { integrativa }\end{array}$ \\
\hline 2017 & $\begin{array}{l}\text { International } \\
\text { Nursing Congress }\end{array}$ & $\begin{array}{ll}\text { ENDRINGER, } & \text { Deyvid } \\
\text { Dantas; } & \text { CRUZ, } \\
\text { Monielle Lima } & \end{array}$ & $\begin{array}{l}\text { Representatividade do } \\
\text { enfermeiro na assistência a } \\
\text { gestantes com pré-eclâmpsia }\end{array}$ \\
\hline 2015 & $\begin{array}{lr}\text { REBES - Revista } \\
\text { Brasileira } & \text { de }\end{array}$ & $\begin{array}{l}\text { MELO, } \\
\text { Ferreira, et al }\end{array}$ & $\begin{array}{l}\text { A hipertensão gestacional e o } \\
\text { risco de pré-eclampsia: }\end{array}$ \\
\hline
\end{tabular}

RC: 97235

Disponível em: https://www.nucleodoconhecimento.com.br/saude/riscos-da- 
Educação e Saúde

2018 Brazilian Journal of OLIVEIRA, Leilyanne Surgery and Clinical de Araújo Mendes, et Research-BJSCR al

\section{Revista Científica SANTOS, Keilane Multidisciplinar Carvalho. \\ Núcleo do \\ Conhecimento}

2016 Revista Científica SANTOS, Joelma da FASETE

\section{0}

FASET

Thiago Paulo de

Almeida

SARMENTO,

Silva, et al revisão bibliográfica

Cuidados de enfermagem a gestante com síndrome hipertensiva: revisão integrativa.

Enfermagem e os cuidados Emergenciais na doença hipertensiva específica na gravidez

Atuação do enfermeiro na redução da ocorrência da préeclâmpsia: uma revisão integrativa

Pré-eclâmpsia na gestação: ênfase na assistência de enfermagem

2016 Centro Universitário TEIXEIRA, Rafaela da Cuidados de enfermagem à Ritter dos Reis

Rosa; MINUZZI, pacientes com hipertensão Renata Nolibus; gestacional: um relato de TELES, Jéssica experiência

Machado

2017 Rev Port Med Geral FERREIRA, Sara Ácido acetilsalicílico na Fam Santos, et al

2015 Ciência Y KRAUZER, Ivete Enfermeria Maroso, et al. prevenção da pré-eclâmpsia: uma revisão baseada na evidência

Sistematização da assistência de enfermagem na atenção básica: 0 que dizem os enfermeiros?

2015 J. res.: fundam. FERNANDES, Amélia Sistematização da assistência

Disponível em: https://www.nucleodoconhecimento.com.br/saude/riscos-da- 
care.

Carolina Lopes

\section{5} Revista Enfermagem

2016 Rev. Interd
MONTEIRO, M. M., et al. de enfermagem na prevenção de infecções em unidade de terapia intensiva

Mirelle Sistematização da assistência de enfermagem: facilidades e desafios do enfermeiro na gerência da assistência

Emergências obstétricas: características de casos atendidos por serviço móvel de urgência

Fonte: Própria autoria (2021)

\subsection{CARACTERÍSTICAS DA HIPERTENSÃO NA GRAVIDEZ}

Nesta pesquisa teremos ênfase em uma patologia que é a segunda maior causa de morte perinatal do Brasil, que é a hipertensão na gravidez, no qual acarreta vários riscos para a mãe e filho, sendo assim necessário o acompanhamento regular desses pacientes e a orientação das mudanças no estilo de vida e na alimentação (ARAÚJO et al., 2017).

A pressão arterial (PA) apresenta alteração diante da interação de fatores neurohumorais, comportamentais e ambientais. A hipertensão arterial (HA) pode ser conceituada como uma síndrome de origem multifatorial, considerada como um dos grandes problemas na área de saúde pública sendo avaliada como grave fator de risco para as doenças cardiovasculares (ARNALDO; CARDOZO, 2021).

As mulheres que fazem uso de cigarro e anticoncepcional, com idade superior a 30 anos, acabam sendo as mais atingidas, pelo fato delas estarem submissas a outras doenças pulmonares. As mulheres recebem proteção devido a eventos 
cardiovasculares que ocorrem antes da menopausa, no homem somente surge depois dos 30 anos (SOARES et al., 2015).

Estudos com prazo mais longo passaram a identificar que elas não são diferentes dos homens quanto à resposta pressórica anti-hipertensiva; porém, é de suma importância evitar o uso de inibidores da Enzima Conversora da Angiotensina (ECA) e de antagonistas do receptor da angiotensina II nas mulheres quando estiverem em idade fértil e que não estejam fazendo uso de métodos anticonceptivos comprovadamente seguros (SANTOS; NETO, 2016).

As causas da hipertensão estão associadas a uma vida sedentária, com a ingestão inadequada de alimentos, como o excesso de sódio e gordura ou com consumo de bebidas alcoólicas, e até mesmo o fumo, que é o causador da insuficiência cardíaca. O fato é que a hipertensão arterial é um potente contribuidor para doenças cardiovasculares, atacando e sobrecarregando o coração até que um dia ele falha (CABRAL, 2019).

A hipertensão na gravidez é a segunda causa de morte materna e perinatal no Brasil segundo dados do ministério da saúde. Isso mostra a necessidade de conhecer e controlar esta patologia. $\mathrm{Na}$ gestação o organismo da mulher passa por várias transformações para poder desenvolver o feto que será abrigado por cerca de 280 dias ou aproximadamente 9 meses. No Brasil a expectativa que a população atingirá a marca de 228 milhões de habitantes em 2025 (CABRAL, 2019).

A taxa de mortalidade segundo o IBGE no ano de 2016, é de que 6,6 nascidos vivos a cada mil habitantes. Diante destes dados, fica evidente a necessidade desenvolver pesquisa e projeto para melhor este índice tão alarmante, a partir do mapeamento dos locais que tem o maior risco gestacionais, que poderá intensificar os cuidados com as mulheres gestante e assim melhora a taxa de mortalidade (ENDRINGER; CRUZ, 2017). 


\subsection{ASPECTOS GERAIS DA PRÉ-ECLÂMPSIA}

Não se sabe ao certo quando foram identificados os primeiros casos dessa patologia, porém o que se sabe, é que ela foi descrita desde os tempos em que os registros começaram a ser feitos, sua ocorrência foi atribuída a maus espíritos, mau gênio, e, mais recentemente, à ingestão de sódio e ao ganho de peso durante a gestação (ENDRINGER; CRUZ, 2017).

Percebe-se que esta síndrome tem causado grandes transtornos em gestantes jovens correlacionados à primeira gestação, sendo que se caracteriza como a principal causa de mortalidade materna no Brasil. Em 1998 a toxemia gravídica foi responsável por $21 \%$ dos óbitos obstétricos diretos (SANTOS; BRASILEIRO, 2018).

A pré-eclâmpsia se caracteriza como uma das síndromes hipertensivas que podem acometer mulheres em período de gravidez, durante o parto e/ou no período de pósparto até 10 dias, de forma que ainda se constitui como uma patologia que quando não adequadamente diagnosticada e tratada, pode causar grandes prejuízos e até levar à morte da gestante e/ou parturiente (SARMENTO et al., 2020).

Ela se caracteriza como uma doença sistêmica que surge a partir da $20^{\underline{a}}$ semana de gestação pela tríade: hipertensão, edema e proteinúria podendo ter seu quadro agravado para eclampsia, na presença de convulsão, e/ou evoluir para síndrome HELLP (SANTOS; BRASILEIRO, 2018).

As causas da pré-eclâmpsia ainda são desconhecidas, porém, o que se sabe é que algumas gestantes apresentam maior propensão de serem acometidas por esta patologia. $\mathrm{Na}$ antiguidade referiam-se aos maus gênios, o ganho de peso, e a ingesta de sal em demasia (FERREIRA et al., 2017).

Hoje ainda não se conhece ao certo os motivos causadores e desencadeadores da pré-eclâmpsia, o que se sabe é que ela tem multicausais: pré-disposição familiar e genética, endócrino-metabólicas, incluindo alteração da produção das prostaglandinas, isquemia uteroplacentário, imunológicas, presença de tecido

RC: 97235

Disponível em: https://www.nucleodoconhecimento.com.br/saude/riscos-dahipertensao 
trofoblástico, toxinas na corrente sanguínea materna, ocasionando vasoconstrição e placenta defeituosa (SARMENTO et al., 2020).

A pré-eclâmpsia tem diversas etiologias podendo provir de causa hereditária e/ou adquiridas devido ao estilo de vida da paciente ou de suas condições sociais e ambientais. Porém, o que se tem conhecimento é que as condições sociais, juntamente com uma vida sedentária, má alimentação, e a falta de informação normalmente proveniente da baixa escolaridade, favorecem o risco eminente do aparecimento da pré-eclâmpsia, além de outras (TEIXEIRA; MINUZZI; TELES, 2016).

A Síndrome Hipertensiva inicialmente se apresenta como pré-eclâmpsia leve, depois evolui para pré-eclâmpsia grave, eclampsia, síndrome de Hellp, CIVD (coagulação intravascular disseminada) e por último óbito. E são estes os estágios de gravidades da síndrome hipertensiva, porém, não necessariamente ela precisa passar por todos os estágios, podendo evoluir de uma pré-eclâmpsia leve para Síndrome Hellp (FERREIRA et al., 2017).

Outras complicações da pré-eclâmpsia são: o descolamento prematuro da placenta, em consequência de lesão placentária no sítio da implantação, o edema agudo de pulmão, a insuficiência renal aguda, em decorrência da glomerulopatia instalada e a hemorragia cerebral (TEIXEIRA; MINUZZI; TELES, 2016).

Os radicais livres de $\mathrm{O} 2$ identifica-se com as células de gordura que são o endotélio, solúvel no sangue materno e muito próximo do endotélio vascular. Reduzem a produção do prostaciclina e óxido nítrico que são vasos dilatados, e aumentam a resistência vascular, causando o aumento da pressão arterial (FERREIRA et al., 2017).

A única alternativa para controlar a pré-eclâmpsia e impedir que evolua para eclampsia é o correto acompanhamento do pré-natal no período gestacional. Pacientes com pré-eclâmpsia leve precisam optar pelo repouso, medir sempre a 
frequência da pressão arterial e aderir a uma dieta com pouco sal (KRAUZ et al., 2015).

Os medicamentos anti-hipertensivos e anticonvulsivantes são indicados para o controle dos quadros de eclampsia com maior gravidade, que podem exigir a antecipação do parto. $\mathrm{E}$ a doença tende a regredir espontaneamente com a retirada da placenta (FERNANDES, 2015).

Diante disso, de acordo com Varella (2020, p. 1), é de sum importância seguir algumas recomendações:

- Se dirigir ao ginecologista antes de engravidar para uma avaliação clínica e início da administração de ácido fólico;

- Importante estar presente em todas as consultas previstas no prénatal;

- Qualquer desatenção e a ausência de sintomas podem fazer com que uma forma leve de pré-eclâmpsia evolua com complicações;

- Realizar exercícios físicos de acordo com a fase da gestação;

- Reduzir a quantidade de sal nas refeições, não fumar e principalmente não ingerir álcool durante a gravidez (VARELLA, 2020, p. 1).

O tratamento consiste em repouso no leito, (lado esquerdo), em local arejado e tranquilo, com a utilização de fármacos, com o intuído de minimizar o agravamento e adiar o trabalho de parto prematuro, quando o possível, ou seja, quando não há risco iminente para a mãe e o feto. Porém quando isso não for possível a indicação é a interrupção imediata da gestação, independente do período gestacional (FERREIRA et al., 2017).

\subsection{A ASSISTÊNCIA DE ENFERMAGEM NOS NÍVEIS DE ATENÇÃO À SAÚDE DA MULHER}

A Sistematização da Assistência de Enfermagem (SAE) visa um cuidado de forma ampla, dinâmica, com planejamento envolvendo os processos de enfermagem em

RC: 97235

Disponível em: https://www.nucleodoconhecimento.com.br/saude/riscos-da-

hipertensao 
seu todo, cujo cuidado conta com a participação harmônica de vários profissionais juntamente com a família e o paciente, construindo um ambiente propicio à recuperação com sucesso e com menos desgaste tanto para o paciente-família como também para a equipe (SOARES et al., 2015).

Os cuidados de enfermagem visam também a integração em sociedade, onde o seu foco principal é proporcionar qualidade de vida às pessoas, de forma a conduzir a um melhor e mais amplo atendimento de suas necessidades especificas, priorizando os indivíduos que se encontram sob atenção e cuidados de enfermagem, ou seja, aqueles que já fazem acompanhamento (MONTEIRO et al., 2015).

A "Enfermagem Planejada" procura realizar a orientação profissional no que concerne a elaboração de um planejamento sistemático da assistência de enfermagem. Atribui-se à série de princípios coordenados entre si aqui expressos a nomenclatura de "Plano Terapêutico de Enfermagem", plano este que compõe todo o processo de ações e decisões destinadas a programar os cuidados de enfermagem. (SOARES et al., 2015, p. 50).

Entende-se que a assistência de enfermagem é todo cuidado ou técnica dispensada a um paciente, e todo procedimento que diminuía ou minimizava a dor. Quando o enfermeiro cuida de um paciente ele dispensa não só uma atenção, mas se liga ao cliente para diminuir suas dores, proporcionando desta forma sua recuperação, diminuindo seus agravos e incentivando sua melhora tanto na parte física como mental, através da ação em conjunto com a equipe de saúde e a família (MONTEIRO, et al., 2015).

Todavia, realizar o planejamento sobre os cuidados de enfermagem é uma missão que apresenta certo grau de complexidade devido a vários fatores: uma destas é que o processo de planejar em enfermagem envolve a participação harmônica de várias pessoas, como o paciente, o enfermeiro e sua equipe e os membros da equipe de saúde; outra razão é a indispensabilidade do uso do raciocínio, da tomada de decisões, da atuação e da documentação; além destas, outra razão 
preponderante está relacionada a constantes mudanças da vida que afetam até os planos mais aperfeiçoados (SOARES et al., 2015).

Observa-se que para realizar uma boa assistência de enfermagem é necessário levar em consideração diversos fatores, sendo um dos principais, a habilidade do enfermeiro em conduzir o trabalho em conjunto com a equipe de saúde, tendo cuidado com paradigmas do paciente e sua família. Utilizando sua capacidade em transmitir informações e por outro lado saber ouvir, e isso se constitui como componentes básicos para um bom profissional de enfermagem (MONTEIRO et al., 2015).

O enfermeiro assistencialista faz parte de uma equipe multiprofissional que visa ações preventivas e curativas no âmbito da comunidade através da promoção de políticas de saúde visando com isso a diminuição das notificações de agravo, através do conhecimento das causas e riscos aos quais a população está exposta, e ao mesmo tempo, sensibilizando os órgãos públicos para as ações que deverão ser implantadas (KRAUZER, 2015).

Ao realizar a anamnese em um determinado cliente deve-se adotar um olhar atencioso e holístico visando não somente a patologia de base ou aquela que está em evidência, mas também, procurar compreender os motivos pelos quais ela emergiu, a fim de buscar junto ao paciente solução para ele (SOARES et al., 2015).

Nesta coleta de dados o enfermeiro deverá traçar planos de cuidados sistematizados com o intuito da promoção e a recuperação da saúde a qual fora abalada, visando com isso, um diagnóstico correto. Para Carpenito apud Fernandes, et al $(2015$, p. 1.581):

O diagnóstico de enfermagem baseia-se tanto nos problemas reais (voltados para o presente) quanto nos problemas potenciais (voltados para o futuro), que podem ser sintomas de disfunções fisiológicas, comportamentais, psicossociais ou espirituais.

RC: 97235

Disponível em: https://www.nucleodoconhecimento.com.br/saude/riscos-da- 
Entende-se que o diagnóstico de enfermagem se divide em dois momentos: diagnostico real que é caracterizado pelas manifestações definidas já existentes no indivíduo, ou seja, mobilidade física prejudicada, relacionada a lesão em membro inferior. O segundo é o diagnóstico de risco caracteriza-se pelos fatores de riscos aos quais o indivíduo está exposto, vulnerável tanto pelo meio quanto pela sua própria imunidade física, ou seja, pelo risco de infecção relacionado ao tempo de internação (KRAUZER, 2015).

\subsection{A ASSISTÊNCIA E AÇÕES DE ENFERMAGEM NA PREVENÇÃO A PRÉ-ECLAMSIA}

Durante a realização do pré-natal alguns exames são solicitados e por meio destes podem ser detectadas as patologias agudas ou crônicas que são direcionadas ao tratamento para não prejudicar o ciclo gestacional e diminuir as taxas de morbimortalidade materno-infantil (ARNALDO; CARDOZO, 2021).

A consulta pré-natal precisa ocorrer logo no começo da gestação, pois pode-se realizar uma avaliação mais detalhada das condições materna e fetal. Mesmo que a gravidez seja considerada um processo fisiológico normal, podem ocorrer diversos tipos de alterações no corpo da mulher e isso acarreta numa menor distância entre saúde e doença do que quando ela não está grávida. A prevenção ou pelo menos diagnóstico precoce dos sinais anormais, e imediatamente um tratamento efetivo, pode funcionar como estratégia para minimizar diversos tipos de complicações associadas a parturição, não somente durante o período anteparto, como também durante o trabalho de parto (ENDRINGER; CRUZ, 2017).

Sabe-se que o profissional de saúde deve estar atento a qualquer sinal que possa indicar a uma situação que comprometa o pré-natal, por este motivo o enfermeiro deverá realizar todos os passos, além desses a solicitação de exames laboratoriais (SANTOS; BRASILEIRO, 2018).

RC: 97235

Disponível em: https://www.nucleodoconhecimento.com.br/saude/riscos-dahipertensao 
A enfermagem precisa sempre prestar atenção durante todo período gestacional no intuito de que quaisquer ocorrências não planejadas não se manifestem durante este evento. As ações de promoção da saúde e prevenção de riscos durante a gestação precisam ter foco direcionado ao atendimento a esta clientela bem mais específica e que exige todo um cuidado. Nesse contexto, o atendimento pré-natal, conforme preconiza o Ministério da Saúde, funciona como o principal caminho para gerar a segurança que a clientela e os profissionais de saúde precisam ter sobre a consolidação de uma gestação livre de riscos e intercorrências (MONTEIRO et al., 2016).

Nesse viés, a consulta de enfermagem, o acompanhamento da gestante durante 0 pré-natal, a atenção diante de qualquer manifestação clínica fora do planejado e o aconselhamento funcionam como fundamentais e precisam receber o devido monitoramento. Por outro lado, chamam atenção para as complicações ligadas à hipertensão arterial, presença de hemorragias, sangramentos e sinais de préeclâmpsia e eclampsia, como balizadoras das complicações mais profundas e da mortalidade (SANTOS; BRASILEIRO, 2018).

A pré-eclâmpsia tem seu início lento e gradual com grandes complicações para a mãe e para o feto, portanto se faz necessário que haja um pré-natal de boa qualidade que proporcione ações preventivas e a identificação dos casos predisponentes a fim de diagnosticar o mais cedo possível, para iniciar o tratamento profilático. Também deverá ser registrado no prontuário as intercorrências para que estas informações não se percam e facilitem o atendimento futuro (MONTEIRO et al., 2016).

O profissional enfermeiro deverá realizar já na primeira consulta com a mulher gestante, uma anamnese completa obtendo o máximo de informações pertinentes à sua saúde atual e pregressa, além dos antecedentes familiares, e para isso deve solicitar exames de rotina, suporte vitamínico, orientações quanto a sua dieta, preenchimento da sua carteira de gestante, verificar seu cartão de vacinação e agendamento para próxima consulta (ENDRINGER; CRUZ, 2017). 
A assistência de enfermagem no período pré-natal é de suma importância porque monitora e identifica possíveis alterações no seu período inicial, e encaminha para uma avaliação mais apurada em uma unidade de referência, com essa correta tomada de decisão pode fazer a diferença, e proporcionar a gestante maior segurança e caracterizar uma verdadeira assistência preventiva, vale ressaltar que 0 profissional enfermeiro é um protagonista de importância incalculável na assistência pré-natal, porque é justamente ele que detém maior vínculo com as gestantes (SANTOS; BRASILEIRO, 2018).

\section{CONSIDERAÇÕES FINAIS}

O estudo, construído a partir de uma revisão de literatura especializada, permitiu uma análise sobre a prevenção da pré-eclâmpsia na atenção básica, e para tanto, fez um breve histórico sobre a incidência dessa patologia, bem como se procurou seus conceitos e suas características.

Além disso, foi oportuno descrever as contribuições da assistência de enfermagem na Atenção Básica. Diante disso, entende-se que para realizar uma boa assistência de enfermagem é necessário levar em consideração diversos fatores, sendo um dos principais, a habilidade do enfermeiro em conduzir o trabalho em conjunto com a equipe de saúde, tendo cuidado com paradigmas do paciente e sua família. Utilizando sua capacidade em transmitir informações e por outro lado saber ouvir, e isso se constitui como componentes básicos para um bom profissional de enfermagem

A pré-eclâmpsia, embora se constitua como uma patologia ou uma síndrome que pode acometer a mulheres em estado de gestação, ainda se caracteriza como um problema de saúde pública, a despeito de todo o desenvolvimento da ciência, inclusive na área de enfermagem e na área médica, justamente o motivo desencadeador do estudo, o qual parte do problema proposto na introdução, o qual, no entendimento dos pesquisadores, foi tratado em profundidade, de forma que os 
dados obtidos se mostraram consistentes e os resultados deles advindos podem ser generalizados a toda a população pesquisada.

Dessa forma, fazendo uso de artigos que foram encontrados tendo como foco a temática deste estudo, desperta sobre a necessidade de serem feitas muitas outras publicações sobre a prevenção da pré-eclâmpsia. A maior parte dos trabalhos concentra seu foco mais na atuação do enfermeiro na humanização do contato profissional/ usuário no momento de dor aguda ou crônica em serviços de emergência do que na prevenção da pré-eclâmpsia na atenção básica.

Então, quais as ações de enfermagem na Atenção Básica diante dos riscos da hipertensão arterial na gestação? Em resposta a problemática, é valido destacar que o profissional de enfermagem atuante em uma unidade de saúde deve realizar palestras com o intuito de sensibilizar sobre a importância de realizar o pré-natal de forma mais rápida possível para garantir uma gravidez saudável e uma boa evolução do concepto e no trabalho de parto.

Nesse sentido, conclui-se que as principais condutas utilizadas não somente pelo enfermeiro, mais por toda a equipe atuante na atenção básica, devem estar centradas no processo de acolhimento, voltados para as ações que podem ser determinantes na prevenção da pré-eclâmpsia. Assim, é de suma importante a necessidade de publicação de mais trabalhos com foco na prevenção da préeclâmpsia na atenção básica.

\section{REFERÊNCIAS}

ARAÚJO, Isabella Félix Meira, et al. Síndromes hipertensivas e fatores de risco associados à gestação. Rev enferm UFPE on line., Recife, 11(Supl. 10):4254-62, out., 2017.

ARNALDO, Mariany de Freitas. CARDOZO, Maryanne Neuraide Freire. Assistência de enfermagem às gestantes hipertensas na prevenção da prematuridade: revisão

RC: 97235

Disponível em: https://www.nucleodoconhecimento.com.br/saude/riscos-dahipertensao 
bibliográfica. Revista científica multidisciplinar núcleo do conhecimento. Ano 06, Ed. 02, Vol. 02, pp. 108 - 125 . Fevereiro de 2021.

CABRAL, Gustavo Pereira. Cuidados de enfermagem a mulheres com doença hipertensiva específica da gestação: uma revisão integrativa. Trabalho de Conclusão de Curso. Uruguaiana, 2019.

ENDRINGER, Deyvid Dantas; CRUZ, Monielle Lima. Representatividade do enfermeiro na assistência a gestantes com pré-eclâmpsia. International Nursing Congress. May 9-12, 2017.

FERNANDES, Amélia Carolina Lopes. Sistematização da assistência de enfermagem na prevenção de infecções em unidade de terapia intensiva. J. res.: fundam. care. online 2015. out./dez. 6(4):1580-1589.

FERREIRA, Sara Santos, et al. Ácido acetilsalicílico na prevenção da pré-eclâmpsia: uma revisão baseada na evidência. Rev Port Med Geral Fam 2017;33:118-32.

FONSECA, J. J. S. Metodologia da pesquisa científica. Fortaleza: UEC, 2002.

GIL, Antônio Carlos. Como elaborar projetos de pesquisa. 4. ed. São Paulo: Atlas, 2007.

KRAUZER, Ivete Maroso, et al. Sistematização da assistência de enfermagem na atenção básica: o que dizem os enfermeiros? Ciência Y Enfermeria XXI (2), 2015.

LAKATOS, Eva Maria; MARCONI, Marina de Andrade. Fundamentos da metodologia científica. 7. ed. São Paulo: Atlas, 2010.

MELO, Wyara Ferreira, et al. A hipertensão gestacional e o risco de pré-eclampsia: revisão bibliográfica. REBES - Revista Brasileira de Educação e Saúde (Pombal PB, Brasil), v. 5, n. 3, p. 07-11, jul-set, 2015. 
MONTEIRO, M. M., et al. Emergências obstétricas: características de casos atendidos por serviço móvel de urgência. Rev. Interd. v. 9, n. 2, p. 136-144, abr/mai/jun. 2016143.

OLIVEIRA, Leilyanne de Araújo Mendes, et al. Cuidados de enfermagem a gestante com síndrome hipertensiva: revisão integrativa. Brazilian Journal of Surgery and Clinical Research - BJSCR. Vol.23,n.2,pp.159-164 (Jun - Ago 2018).

SANTOS, Keilane Carvalho. BRASILEIRO, Marislei Espíndula. Enfermagem e os cuidados Emergenciais na doença hipertensiva específica na gravidez. Revista Científica Multidisciplinar Núcleo do Conhecimento. Ano 03, Ed. 11, Vol. 08, pp. 1726 Novembro de 2018.

SANTOS, Joelma Oliveira Silva; NETO, Thiago Paulo de Almeida. Atuação do enfermeiro na redução da ocorrência da pré-eclâmpsia: uma revisão integrativa. Revista Científica da FASETE 2016.1.

SARMENTO, Rayani Silva, et al. Pré-eclâmpsia na gestação: ênfase na assistência de enfermagem. Enfermagem Brasil 2020;19(3):261-267.

SILVA, E.L. da; MENEZES, E.M. Metodologia da pesquisa e elaboração de dissertação. Florianópolis: Laboratório de Ensino à Distância da UFSC, 2001.

SOARES, Mirelle Inácio, et al. Sistematização da assistência de enfermagem: facilidades e desafios do enfermeiro na gerência da assistência. Escola Anna Nery Revista de Enfermagem 19(1) Jan-Mar 2015.

TEIXEIRA, Rafaela da Rosa; MINUZZI, Renata Nolibus; TELES, Jéssica Machado. Cuidados de enfermagem à pacientes com hipertensão gestacional: um relato de experiência. XII Semana de Extensão, Pesquisa e Pós-Graduação SEPesq - 24 a 28 de outubro de 2016

Enviado: Agosto, 2021. 
Aprovado: Setembro, 2021. 